\title{
Activity of Plant Extracts/Essential Oils Against Three Plant Pathogenic Fungi and Mosquito Larvae: GC/MS Analysis of Bioactive Compounds
}

\author{
Younis K. Hamad, ${ }^{\mathrm{a}, \mathrm{b}}$ Yasser Abobakr, ${ }^{\mathrm{a}, \mathrm{c}}$ Mohamed Z. M. Salem, ${ }^{\mathrm{d}, *}$ Hayssam M. Ali, ${ }^{\text {e,f }}$ \\ Ali S. Al-Sarar, ${ }^{a}$ and Ali A. Al-Zabib ${ }^{a}$
}

\begin{abstract}
Certain natural products extracted from different parts of medicinal and aromatic plants were examined for their antifungal activity against three plant pathogenic fungi, Fusarium oxysporum, Rhizoctonia solani, and Alternaria solani, and insecticidal activity against mosquito larvae (Culex pipiens). Acetone extract of Tectona grandis showed the highest antifungal activity against $R$. solani and $A$. solani with $\mathrm{EC}_{50}$ values of 118 and $294 \mu \mathrm{g} / \mathrm{mL}$, respectively. The highest larvicidal activity was displayed by the essential oils of Ocimum basilicum and Eucalyptus gomphocephala with $\mathrm{LC}_{50}$ value of 22 , and $30 \mathrm{mg} / \mathrm{L}$, respectively. By gas chromatographymass spectrometry (GC/MS) analysis 3-allylguaiacol (65.8\%) and eugenol acetate $(46.6 \%)$ were the main compounds in Syzygium aromaticum methanolic extract and essential oil, respectively. The main compound in $T$. grandis acetone extract was cyclohexylpentyl oxalate (8.7\%); its water extract contained $(E)$-4,4-dimethyl-2-pentene (51.1\%); E. gomphocephala branch oil contained $p$-cymene (28.8\%); Euphorbia paralias leaf extract contained $1 \beta \mathrm{H}$-romneine (26.3\%); the seed extract contained $\alpha$-linolenic acid, TMS (15.2\%); Punica granatum extract contained furfural (32.1\%); and $O$. basilicum essential oil contained estragole (65.9\%). Thus, extracts from the tested plants can be used as natural biofungicides to manage diseases caused by F. oxysporum, $R$. solani, and A. solani. Additionally, these extracts show potential la rvicide activities against mosquito larvae.
\end{abstract}

Keywords: Antifungal activity; Larvicidal activity; Pathogenic fungi Natural extract; Essential oils

Contact information: a: Department of Plant Protection, College of Food and Agriculture Sciences, King Saud University, P.O. Box 2460, Riyadh 11451, Saudi Arabia; b: Plant Pathology Department, Faculty of Agriculture, Alexandria University, Alexandria, Egypt; c: Plant Protection Research Institute, Sabahia Research Station, Agricultural Research Center, Alexandria, Egypt; d: Forestry and Wood Technology Department, Faculty of Agriculture (EL-Shatby), Alexandria University, Alexandria, Egypt; e: Botany and Microbiology Department, College of Science, King Saud University, P.O. Box 2455, Riyadh 11451, Saudi Arabia; f: Timber Trees Research Department, Sabahia Horticulture Research Station, Horticulture

Research Institute, Agriculture Research Center, Alexandria, Egypt;

*Corresponding author:_zidan_forest@yahoo.com; mohamed-salem@alexu.edu.eg

\section{INTRODUCTION}

In crop plants, fungi cause more economic damage than any other group of microorganisms, with annual losses estimated at more than $\$ 200$ billion (Horbach et al. 2011). Chemical control of phytopathogenic fungi efficiently reduces the negative consequences resulting from these organisms; however, field application of these chemicals is not desirable. Extensive and improper use of synthetic fungicides can lead to serious consequences on the environment and on the health of humans and animals. Moreover, the development of fungicide-resistance in phytopathogenic fungi has been 
reported (Hahn 2014). Therefore, the search for novel antifungal agents is needed. Plantderived products may be an alternative approach against phytopathogenic fungi (Amadi et al. 2010; Badawy and Abdelgaleil 2014).

p-cymene and crypton were found to be the main compounds of leaf essential oil (EO) from Eucalyptus gomphocephala growing in Egypt (Salem et al. 2015). 1,8-cineole, pinene, viridiflorol, terpineol, aromadendrene and trans-pinocarveol are the abundant compounds in the leaf EO of E. procera cultivated in central Iran (Rahimi-Nasrabadi et al. 2012), while 1,8-cineole, cryptone, 4-allyloxyimino-2-carene, and 4-terpineol were found in leaf EO from E. largiflorens from the same area (Rahimi-Nasrabadi et al. 2013) with high antimicrobial activities. Also, 1,8-cineole, $\alpha$-pinene, and $\alpha$-terpineol are the main constituents of E. oleosa leaf EO with high antibacterial activity (Rahimi-Nasrabadi et al. 2013).

Different bioactive compounds of naphtoquinone, anthraquinone, 2-methyl anthraquinone (techtoquinone), lapachol, and deoxylapachol have been isolated from teak wood extracts (Windeisen et al. 2003; Thulasidas and Bhat 2007). Several studies have shown antifungal efficacy of plant extracts and EOs against plant and human pathogenic fungi (Mahboubi and Bidgoli 2010; Salem et al. 2016a,b; Sales et al. 2016). For example, the antifungal activity of natural extracts and EOs derived from Tectona grandis, Syzygium aromaticum, and Eucalyptus gomphocephala were tested against Fusarium moniliforme, Fusarium oxysporum, Aspergillus sp., Mucor sp., and Arthrinium phaeospermum. These compounds inhibit both the mycelial growth and sporulation of fungi (Astiti and Suprapta 2012). Moreover, the EO obtained from E. camaldulensis completely inhibits the mycelial growth of the five isolates of Fusarium spp. at a concentration range between 7 and 8 $\mu \mathrm{L} / \mathrm{mL}$ after five days of incubation (Gakuubi et al. 2017).

Mosquitoes are insects that cause great concern for public health, as they are vectors for numerous tropical and subtropical diseases. They are an important threat for over two billion people in the world (Odalo et al. 2005). The intensive use of conventional insecticides to control mosquitoes is causing many problems such as environmental pollution, toxic hazards to mammals and non-target organisms, and the development of insecticide resistance (Sutthanont et al. 2010). These complications have become the driving force for an expeditious search for alternatives: compounds offering protection against mosquitoes suitable for both public health and the environment. Among the present alternative approaches aimed at reducing mosquito populations, the use of biopesticides based on natural plant products is now one of the most promising (Rajamma et al. 2011).

The present study investigated the antifungal activity of natural extracts and EOs obtained from six aromatic plants, T. grandis, E. gomphocephala, S. aromaticum, Euphorbia paralias, Ocimum basilicum, and Punica granatum against three plant pathogenic fungi, F. oxysporum, Rhizoctonia solani, and Alternaria solani. These extracts also were tested for their efficacy against mosquitoes. Lastly, the chemical constituents of the extracts/EOs were identified using GC/MS analysis.

\section{EXPERIMENTAL}

\section{Plant Materials and Their Solvent Extraction}

The plant materials used in this study are presented in Table 1. The plant materials used for solvent extraction were previously air-dried under room temperature for one week and then ground to fine particles (40- to 60-mesh). Approximately $100 \mathrm{~g}$ of ground material 
was soaked in solvent ( $200 \mathrm{~mL}$ ) for 3 days, filtered, and concentrated to dryness using a rotary evaporator. The essential oils (EOs) were extracted by hydrodistillation using a Clevenger-type apparatus, where $100 \mathrm{~g}$ of green materials was hydrodistillated for $3 \mathrm{~h}$. The collected oil was stored at $4{ }^{\circ} \mathrm{C}$ prior to analysis (Salem et al. 2013).

Table 1. Plant Materials and Their Parts Used for the Extractions

\begin{tabular}{|c|c|c|}
\hline Plant & Part used & Extract/EO \\
\hline Tectona grandis & Air dried wood & Acetone extract \\
\hline T. grandis & Air dried wood & Water extract \\
\hline Eucalyptus gomphocephala & Green branches & EO \\
\hline Syzygium aromaticum & Air dried flower buds & Methanolic extract \\
\hline S. aromaticum & Air dried flower buds & EO \\
\hline Euphorbia paralias & Air dried leaves & Acetone extract \\
\hline E. paralias & Air dried seeds & Acetone extract \\
\hline Ocimum basilicum & Green leaves & EO \\
\hline Punica granatum & Air dried peels & Acetone extract \\
\hline
\end{tabular}

\section{Antifungal Assay}

The antifungal activities of the plant extracts and/or EOs obtained from $T$. grandis, E. gomphocephala, S. aromaticum, E. paralias, O. basilicum, and $P$. granatum were evaluated on the mycelial growth of plant pathogenic fungi (Fusarium oxysporum, Rhizoctonia solani, and Alternaria solani) using the radial growth technique method (Zambonelli et al. 1996). The fungi were obtained from the Microbiological Laboratory, Department of Plant Protection, College of Food and Agriculture Sciences, King Saud University. The plant extracts dissolved in dimethyl sulfoxide (DMSO) were added to warm PDA medium ( 40 to $45^{\circ} \mathrm{C}$ ) at different concentrations, ranging from 50 to 1000 $\mathrm{mg} / \mathrm{L}$, before immediately pouring into $9-\mathrm{cm}$ Petri dishes. Each concentration was tested in triplicate. Parallel controls contained PDA mixed only with DMSO. Mycelial discs (0.5 $\mathrm{cm}$ in diameter) of the plant pathogenic fungi, taken from 8-day-old cultures on PDA dishes, were transferred to the center of PDA dishes supplemented with either plant extracts or only DMSO. Inoculated dishes were incubated at $25^{\circ} \mathrm{C}$ in the dark. The colony growth diameter was measured when the fungal growth in the control treatments had completely covered the Petri dishes. Percentage of mycelial growth inhibition was calculated using the following equation (Pandey et al. 1982),

$$
\text { Mycelial growth inhibition }(\%)=[(\text { DC-DT }) / D C] \times 100
$$

where DC and DT are average diameters of the fungal colony of control and treatment, respectively. The concentrations of plant extract or oil that inhibited the fungi mycelial growth by $50 \%$ ( $\left.\mathrm{EC}_{50}\right)$ with an equivalent confidence limit of $95 \%$ were estimated by the probit analysis method (Finney 1971).

\section{Mosquitoes}

A laboratory colony of Culex pipiens L. (Diptera: Culicidae) obtained from the College of Food and Agriculture Sciences, King Saud University was used. Mosquitoes were maintained at $25 \pm 2{ }^{\circ} \mathrm{C}, 60 \pm 5 \% \mathrm{RH}$, and a 12-h photoperiod. Adult mosquitoes were provided with a $10 \%$ sucrose solution as food. A blood meal was introduced twice per week to feed the females. Larvae were reared in $\mathrm{Cl}$-free water and fed daily with rabbit feed.

Hamad et al. (2019). "Bioactive natural products," BioResources 14(2), 4489-4511. 


\section{Larvicidal Bioassay}

The larvicidal activity of the extracts or oils was evaluated on the early fourth instar of $C$. pipiens larvae using the standard method described by the World Health Organization (WHO 1981), with slight modification. Stock solutions of the extracts or oils were prepared in ethanol, and Tween-80 (10 ppm) was used as an emulsifier. A series of six concentrations of each extract or EO were prepared in dechlorinated tap water. Twenty mosquito larvae were put into $200 \mathrm{~mL}$ cups containing $100 \mathrm{~mL}$ of test solution. The control treatment was prepared with water containing the same concentration of ethanol and Tween-80. Three replicates were used for each concentration. Larval mortalities were recorded after $24 \mathrm{~h}$ of exposure. Larvae were considered dead when they did not respond to probing with a needle. Mortality data were subjected to probit analysis to calculate the median lethal concentration values $\left(\mathrm{LC}_{50}\right)$ of the extracts or oils (Finney 1971). Abbot's formula (Abbott 1925) was used when necessary to correct percentage mortality in the treatments.

\section{GC/MS Analysis of Extracts/Essential Oils}

Samples of $T$. grandis (wood acetone and water extracts) and E. paralias (leaf and seed extracts) were analyzed using a derivatization process. For the derivatization process, $10 \mu \mathrm{L}$ from the samples was added to the bottom of a $2 \mathrm{~mL}$ tube and dried with a gentle stream of pure nitrogen gas (99.999\%). Immediately, $50 \mu \mathrm{L}$ of $\mathrm{N}, O$-Bis (trimethylsilyl, TMS) trifluoroacetamide (BSTFA) was added and vortexed. The reaction was incubated for $3 \mathrm{~h}$ at $70{ }^{\circ} \mathrm{C}$. The products were dried by nitrogen blowing. After drying, hexane was added for dilution and injection into the GC-MS instrument. The injection volume was 2 $\mu \mathrm{L}$ in splitless mode. The analysis of the alteration products and external standards was carried out by GC-MS on a Hewlett- Packard 6890 GC coupled to a 5973 Mass Selective Detector using a DB-5 (J and W Scientific, Agilent, Palo Alto, CA, USA) fused silica capillary column (30 $\mathrm{m} \times 0.25 \mathrm{~mm}$ i.d., $0.25 \mu \mathrm{m}$ film thickness) and helium as a carrier gas. The GC was temperature programmed from $65{ }^{\circ} \mathrm{C}$ ( 2 min initial time) to $300{ }^{\circ} \mathrm{C}$ at 6 ${ }^{\circ} \mathrm{C} / \mathrm{min}$ (isothermal for $20 \mathrm{~min}$ final time). The MS was operated in the electron impact mode at $70 \mathrm{eV}$ ion source energy. Data were acquired and processed with a HewlettPackard Chemstation and compounds were identified by comparison of mass spectra with those of authentic standards, literature and library data, and characterized mixtures. Unknown compounds were characterized by interpretation of the fragmentation pattern of their mass spectra.

The chemical constituents of $S$. aromaticum (methanolic extract and EO), E. gomphocephala (branch oil), P. granatum (extract), and $O$. basilicum (essential oil) were analyzed for their chemical compositions using the previous published methods (Salem et al. 2016a,b, 2019). Identification of the chemical composition of extracts/EOs was done based on MS library searches (NIST and Wiley), as well as by comparing with the MS literature data (NIST 11. 2011; Oberacher 2011).

\section{RESULTS AND DISCUSSION}

\section{Antifungal Activity}

The antifungal activities of plant extracts/EO obtained from different plants against $R$. solani, A. solani, and $F$. oxysporum in terms of radial growth inhibition are summarized in Tables 2, 3 and 4, respectively. The acetone extract of $T$. grandis showed the highest antifungal activity against $R$. solani and $A$. solani with $\mathrm{EC}_{50}$ values of 118 and $294 \mu \mathrm{g} / \mathrm{mL}$, 
respectively. However, the acetone extract of $T$. grandis showed the lowest antifungal activity against $F$. oxysporum. $S$. aromaticum extract exhibited moderate antifungal activities against the three tested fungi. Generally, $F$. oxysporum was less sensitive to $T$. grandis and E. gomphocephala extracts compared with the other two fungi. $E$. gomphocephala branch EO showed weaker antifungal activity than $T$. grandis and $S$. aromaticum extracts. Extracts of E. paralias (leaves), E. paralias (fruits), O. basilicum, T. grandis (obtained from wood), and $P$. granatum exhibited no antifungal activity against the three tested fungi.

Table 2. In vitro Antifungal Activity of Plant Extracts/Oils against $R$. solani using the Mycelial Growth Inhibition Method

\begin{tabular}{|c|c|c|c|c|c|c|}
\hline \multirow[t]{2}{*}{ Extract/EO } & \multirow{2}{*}{$\begin{array}{c}E_{50} \\
(\mu \mathrm{g} / \mathrm{mL})\end{array}$} & \multicolumn{2}{|c|}{ 95\% Confidence limits } & \multirow[t]{2}{*}{ Slope \pm SE } & \multirow[t]{2}{*}{$\mathrm{Chi}^{2}$} & \multirow[t]{2}{*}{$r$} \\
\hline & & Lower & Upper & & & \\
\hline $\begin{array}{l}\text { T. grandis wood acetone } \\
\text { extract }\end{array}$ & 118.39 & 32.57 & 206.41 & $0.69 \pm 0.18$ & 0.17 & 0.99 \\
\hline $\begin{array}{c}\text { E. gomphocephala branch } \\
\text { EO }\end{array}$ & 446.17 & 355.40 & 518.16 & $3.36 \pm 0.62$ & 1.6 & 0.98 \\
\hline $\begin{array}{l}\text { S. aromaticum methanolic } \\
\text { flower buds' extract }\end{array}$ & 491.22 & 467.95 & 520.37 & $8.53 \pm 1.14$ & 0.57 & 0.99 \\
\hline $\begin{array}{l}\text { S. aromaticum flower buds' } \\
\text { EO }\end{array}$ & $<1000$ & & & & & \\
\hline $\begin{array}{c}\text { E. paralias acetone leaf } \\
\text { extract }\end{array}$ & $<1000$ & & & & & \\
\hline $\begin{array}{c}\text { E. paralias seeds acetone } \\
\text { extract }\end{array}$ & $<1000$ & & & & & \\
\hline O. basilicum leaf EO & $<1000$ & & & & & \\
\hline $\begin{array}{c}\text { T. grandis wood water } \\
\text { extract }\end{array}$ & $<1000$ & & & & & \\
\hline $\begin{array}{c}P . \text { granatum peels acetone } \\
\text { extract }\end{array}$ & $<1000$ & & & & & \\
\hline
\end{tabular}

Table 3. In vitro Antifungal Activity of Plant Extracts/Oils against $A$. solani using the Mycelial Growth Inhibition Method

\begin{tabular}{|c|c|c|c|c|c|c|}
\hline \multirow[t]{2}{*}{ Extract/EO } & \multirow[t]{2}{*}{$\begin{array}{c}E_{50} C_{50} \\
(\mu \mathrm{g} / \mathrm{mL})\end{array}$} & \multicolumn{2}{|c|}{$\begin{array}{l}95 \% \text { Confidence } \\
\text { limits }\end{array}$} & \multirow[t]{2}{*}{ Slope \pm SE } & \multirow[t]{2}{*}{$\mathrm{Chi}^{2}$} & \multirow[t]{2}{*}{$r$} \\
\hline & & Lower & Upper & & & \\
\hline $\begin{array}{c}\text { T. grandis wood acetone } \\
\text { extract }\end{array}$ & 294.12 & 168.74 & 457.58 & $0.78 \pm 0.18$ & 0.22 & 0.99 \\
\hline E. gomphocephala branch EO & 991.31 & 847.49 & 1262.4 & $2.9 \pm 0.50$ & 0.07 & 0.99 \\
\hline $\begin{array}{l}\text { S. aromaticum methanolic } \\
\text { flower buds' extract }\end{array}$ & 635.57 & 561.11 & 713.25 & $3.7 \pm 0.52$ & 0.02 & 0.99 \\
\hline $\begin{array}{c}\text { S. aromaticum flower buds' } \\
\text { EO }\end{array}$ & $<1000$ & & & & & \\
\hline $\begin{array}{c}\text { E. paralias acetone leaf } \\
\text { extract }\end{array}$ & $<1000$ & & & & & \\
\hline $\begin{array}{c}\text { E. paralias acetone seeds } \\
\text { extract }\end{array}$ & $<1000$ & & & & & \\
\hline O. basilicum leaf EO & $<1000$ & & & & & \\
\hline T. grandis wood water extract & $<1000$ & & & & & \\
\hline $\begin{array}{c}P . \text { granatum peels acetone } \\
\text { extract }\end{array}$ & $<1000$ & & & & & \\
\hline
\end{tabular}


Table 4. In vitro Antifungal Activity of Plant Extracts/Oils against $F$. oxysporum using the Mycelial Growth Inhibition Method

\begin{tabular}{|c|c|c|c|c|c|c|}
\hline & \multirow{2}{*}{$\begin{array}{c}\mathrm{EC}_{50} \\
(\mu \mathrm{g} / \mathrm{mL})\end{array}$} & \multicolumn{2}{|c|}{$\begin{array}{c}95 \% \text { Confidence } \\
\text { limits }\end{array}$} & Slope $\pm \mathrm{SE}$ & Chi $^{2}$ & $r$ \\
\cline { 3 - 6 } & & Lower & Upper & & \\
\hline $\begin{array}{c}\text { T. grandis wood acetone } \\
\text { extract }\end{array}$ & 965.31 & 641.18 & 2011.2 & $0.93 \pm 0.19$ & 0.18 & 0.99 \\
\hline $\begin{array}{c}\text { E. gomphocephala branch } \\
\text { EO }\end{array}$ & 922.04 & 690.71 & 1416.2 & $1.36 \pm 0.21$ & 2.95 & 0.97 \\
\hline $\begin{array}{c}\text { S. aromaticum flower buds' } \\
\text { extract }\end{array}$ & 439.17 & 416.77 & 460.02 & $9.1 \pm 1.65$ & 0.04 & 0.98 \\
\hline $\begin{array}{c}\text { S. aromaticum flower buds' } \\
\text { EO }\end{array}$ & $<1000$ & & & & & \\
\hline $\begin{array}{c}\text { E. paralias acetone leaf } \\
\text { extract }\end{array}$ & $<1000$ & & & & & \\
\hline $\begin{array}{c}\text { E. paralias acetone seeds } \\
\text { extract }\end{array}$ & $<1000$ & & & & & \\
\hline $\begin{array}{c}\text { O. basilicum leaf EO } \\
\text { T. grandis wood water extract }\end{array}$ & $<1000$ & & & & & \\
\hline $\begin{array}{c}\text { P. granatum peels acetone } \\
\text { extract }\end{array}$ & $<1000$ & & & & & \\
\hline
\end{tabular}

\section{Larvicidal Activity}

The larvicidal activity results of the test plant extracts/EO against $C$. pipiens $\mathrm{L}$. are summarized in Table 5.

Table 5. Larvicidal Activity of Plant Extracts/Oils against Culex pipiens L.

\begin{tabular}{|c|c|c|c|c|c|c|c|}
\hline \multirow[t]{2}{*}{ Plant } & \multirow[t]{2}{*}{ Extract/EO } & \multirow{2}{*}{$\begin{array}{l}\mathrm{LC}_{50} \\
(\mathrm{mg} / \mathrm{L})\end{array}$} & \multicolumn{2}{|c|}{$\begin{array}{l}\text { 95\% Confidence } \\
\text { limits }\end{array}$} & \multirow{2}{*}{$\begin{array}{l}\text { Slope } \pm \\
\text { SE }\end{array}$} & \multirow[t]{2}{*}{$\mathrm{Chi}^{2}$} & \multirow[t]{2}{*}{$r$} \\
\hline & & & Lower & Upper & & & \\
\hline T. grandis wood & $\begin{array}{c}\text { Acetone } \\
\text { extract }\end{array}$ & 251.39 & 138.65 & 329.57 & $\begin{array}{c}3.50 \pm \\
1.08\end{array}$ & 1.22 & 0.95 \\
\hline T. grandis wood & Water extract & 696.74 & 639.36 & 755.41 & $\begin{array}{l}8.13 \pm \\
1.26\end{array}$ & 0.88 & 0.99 \\
\hline $\begin{array}{l}\text { E. gomphocephala } \\
\text { branches }\end{array}$ & $\mathrm{EO}$ & 30.07 & 29.04 & 31.15 & $\begin{array}{c}9.63 \pm \\
0.70\end{array}$ & 2.48 & 0.99 \\
\hline $\begin{array}{l}\text { S. aromaticum } \\
\text { flower buds }\end{array}$ & $\begin{array}{c}\text { Methanolic } \\
\text { extract }\end{array}$ & 58.73 & 48.36 & 70.47 & $\begin{array}{c}1.67 \pm \\
0.19\end{array}$ & 2.50 & 0.98 \\
\hline $\begin{array}{l}\text { S. aromaticum } \\
\text { flower buds }\end{array}$ & EO & 128.92 & 116.46 & 140.07 & $\begin{array}{l}4.16 \pm \\
0.63\end{array}$ & 0.42 & 0.99 \\
\hline E. paralias leaves & $\begin{array}{l}\text { Acetone } \\
\text { Extract }\end{array}$ & 786.44 & 713.09 & 880.48 & $\begin{array}{l}6.02 \pm \\
1.07\end{array}$ & 2.07 & 0.98 \\
\hline E. paralias seeds & $\begin{array}{l}\text { Acetone } \\
\text { Extract }\end{array}$ & 295.45 & 182.41 & 387.39 & $\begin{array}{c}3.38 \pm \\
1.03 \\
\end{array}$ & 2.11 & 0.92 \\
\hline $\begin{array}{l}\text { O. basilicum } \\
\text { leaves }\end{array}$ & EO & 22.00 & 20.59 & 23.41 & $\begin{array}{c}6.49 \pm \\
0.59 \\
\end{array}$ & 1.32 & 0.99 \\
\hline$P$. granatum peels & $\begin{array}{l}\text { Acetone } \\
\text { Extract }\end{array}$ & 955.35 & 807.33 & 1136.1 & $\begin{array}{c}6.18 \pm \\
1.78\end{array}$ & 1.78 & 0.95 \\
\hline
\end{tabular}

The EOs of basil $O$. basilicum and E. gomphocephala displayed the highest larvicidal activity with $\mathrm{LC}_{50}$ values of 22 and $30.1 \mathrm{mg} / \mathrm{L}$, respectively. Moreover, the methanolic extract of $S$. aromaticum showed high larvicidal activity against $C$. pipiens $\left(\mathrm{LC}_{50}=58.7 \mathrm{mg} / \mathrm{L}\right)$, while the essential oil of the same plant showed relatively lower 
larvicidal activity $\left(\mathrm{LC}_{50}=129 \mathrm{mg} / \mathrm{L}\right)$. The acetone extract of $T$. grandis was more toxic to the larvae than the water extract of the same plant with LC 50 values of 251 and 697, respectively. The fruit extract of $E$. paralias exhibited more larvicidal activity to the larvae $\left(\mathrm{LC}_{50}=295 \mathrm{mg} / \mathrm{L}\right)$ than the leaves extract $\left(\mathrm{LC}_{50}=786 \mathrm{mg} / \mathrm{L}\right)$. Low larvicidal activity was observed for $P$. granatum extract with an $\mathrm{LC}_{50}$ value of $955 \mathrm{mg} / \mathrm{L}$.

\section{Chemical Composition of the Extracts/Essential Oils}

3-Allylguaiacol $(65.8 \%)$ and eugenol acetate $(23.4 \%)$ were the main compounds in $S$. aromaticum methanolic extract (Table 6), while eugenol acetate $(46.6 \%)$, isoeugenol $(21.5 \%)$, trans-caryophyllene (15.8\%) and $\alpha$-humulene $(9.0 \%)$ were the main compounds in the EO (Table 7).

Table 6. Chemical Constituents of $S$. aromaticum Methanolic Extract

\begin{tabular}{|c|c|c|c|c|}
\hline Compound name & $\mathrm{RT}^{\mathrm{a}}(\mathrm{min})$ & Area $\%$ & $\mathrm{SI}^{\mathrm{b}}$ & $\mathrm{RSI}^{\mathrm{c}}$ \\
\hline 3-Allylguaiacol & 13.98 & 65.79 & 884 & 922 \\
\hline trans-Caryophyllene & 15.16 & 6.5 & 687 & 846 \\
\hline 1,4,7-Cycloundecatriene & 15.77 & 3.25 & 708 & 904 \\
\hline Eugenol acetate & 16.69 & 23.36 & 701 & 874 \\
\hline Octadecanoic acid & 23.25 & 0.68 & 664 & 751 \\
\hline trans-9-octadecenoic acid & 24.86 & 0.42 & 856 & 868 \\
\hline
\end{tabular}

aRT, Retention Time (min.).

bSI, Standard Index.

${ }^{\mathrm{c}} \mathrm{RSI}$, Reverse Standard index.

Table 7. Chemical Constituents of $S$. aromaticum Essential Oil

\begin{tabular}{|c|c|c|c|c|}
\hline Compound Name & $\mathrm{RT}^{\mathrm{a}}(\mathrm{min})$ & Area \% & $\mathrm{Sl}^{\mathrm{b}}$ & $\mathrm{RSI}^{\mathrm{c}}$ \\
\hline 2-Heptyl acetate & 8.99 & 0.43 & 862 & 933 \\
\hline 2-Nonanone & 9.84 & 0.24 & 858 & 924 \\
\hline linalool & 10.01 & 0.06 & 813 & 866 \\
\hline Benzyl acetate & 10.97 & 0.12 & 858 & 922 \\
\hline 3-Cyclohexen-1-ol & 11.32 & 0.15 & 810 & 905 \\
\hline Cryptone & 11.41 & 0.12 & 809 & 860 \\
\hline Chavicol & 12.34 & 0.12 & 895 & 936 \\
\hline Eugenol acetate & 14.12 & 46.62 & 894 & 911 \\
\hline$\alpha$-Copaene & 14.31 & 0.78 & 823 & 937 \\
\hline trans-Caryophyllene & 15.32 & 15.81 & 823 & 929 \\
\hline$\alpha$-Humulene & 15.87 & 8.96 & 875 & 918 \\
\hline$\alpha-$-Amorphene & 16.10 & 0.26 & 866 & 903 \\
\hline Germacrene-D & 16.26 & 0.29 & 875 & 918 \\
\hline Valencene 1 & 16.41 & 0.05 & 821 & 924 \\
\hline E,E- $\alpha$-Farnesene & 16.48 & 0.71 & 816 & 923 \\
\hline Isoeugenol & 16.76 & 21.46 & 815 & 900 \\
\hline$\alpha$-Cadinene & 16.83 & 0.58 & 913 & 951 \\
\hline Benzeneacetic acid & 17.27 & 0.06 & 909 & 941 \\
\hline [2,2-Dimethyl-4-(3-methylbut-2-enyl)-6- & 17.94 & 1.24 & 896 & 925 \\
methylidene-cyclohexyl]methanol & & & & \\
\hline Caryophylladienol I & 18.71 & 0.12 & 885 & 927 \\
\hline
\end{tabular}

aRT, Retention Time (min.).

bSI, Standard Index.

'RSI, Reverse Standard index. 
Table 8. Chemical Constituents of T. grandis Wood Acetone Extract

\begin{tabular}{|c|c|c|c|c|}
\hline Compound Name & $\mathrm{RT}^{\mathrm{a}}$ (min) & Area (\%) & $\mathrm{SI}^{\mathrm{b}}$ & $\mathrm{RSI}^{\mathrm{c}}$ \\
\hline Vinylather & 5.84 & 2.84 & 773 & 790 \\
\hline 2,2-Dimethyl-3-propyloxirane & 6.29 & 1.05 & 714 & 745 \\
\hline Cyclohexylpentyl oxalate & 6.78 & 8.66 & 735 & 772 \\
\hline 1-Methyl-2,3-dihydro-1H-pyrrole & 7.87 & 0.67 & 734 & 771 \\
\hline $\begin{array}{c}\text { 3-[(2-methoxyethoxy)methoxy]-2- } \\
\text { methyl-tricyclo[5.2.2.0(2,6)]undec-8- } \\
\text { en-11-one }\end{array}$ & 7.95 & 0.97 & 678 & 804 \\
\hline Glycerol, TMS derivative & 14.34 & 0.84 & 747 & 787 \\
\hline Eudesma-3,7(11)-diene & 21.84 & 0.62 & 693 & 823 \\
\hline $\begin{array}{l}\text { 2-Isopropyl-5-methyl-9-methylene- } \\
\text { bicyclo[4.4.0]dec-1-ene }\end{array}$ & 22.11 & 0.58 & 734 & 777 \\
\hline$\alpha$-D-Arabinofuranose, 4TMS derivative & 22.31 & 0.48 & 834 & 863 \\
\hline Phthalic acid, 2TMS derivative & 22.89 & 1.13 & 836 & 867 \\
\hline Vanillic acid, 2TMS derivative & 24.13 & 0.80 & 838 & 865 \\
\hline Protocatechoic acid, 3TMS derivative & 25.17 & 0.75 & 776 & 814 \\
\hline $\begin{array}{c}\text { 2-Thioxo-cis-perhydro-3,1- } \\
\text { benzoxazine }\end{array}$ & 25.57 & 0.39 & 776 & 813 \\
\hline $\begin{array}{l}\text { Methyl- } \alpha \text { - } D \text {-glucofuranoside, } 4 \text { TMS } \\
\text { derivative }\end{array}$ & 25.86 & 0.52 & 765 & 805 \\
\hline 2-Thiobarbituric acid, TMS & 26.20 & 6.58 & 825 & 865 \\
\hline $\begin{array}{c}\text { 3-Hydroxy-3-methylglutaric acid, tri- } \\
\text { TMS }\end{array}$ & 26.27 & 3.21 & 752 & 798 \\
\hline D-Galactose, 5TMS derivative & 26.74 & 0.48 & 778 & 820 \\
\hline Palmitic acid, TMS derivative & 28.65 & 1.08 & 902 & 934 \\
\hline Techtoquinone & 29.72 & 8.46 & 903 & 948 \\
\hline 2-Methylbenzenethiol, TMS derivative & 30.63 & 2.34 & 890 & 933 \\
\hline Stearic acid, TMS derivative & 31.59 & 0.53 & 888 & 924 \\
\hline $\begin{array}{l}\text { 3-(1,3-Benzodioxol-5-yl)-5-hydroxy-4- } \\
\text { nitrocyclohexanone }\end{array}$ & 34.93 & 7.23 & 886 & 910 \\
\hline 1,2-bis(TMS-oxy)-cyclooctene & 35.36 & 8.58 & 913 & 915 \\
\hline Cyclohexyl isobutyl phthalate & 35.73 & 0.71 & 924 & 945 \\
\hline trans-Crotyl alcohol, TMS derivative & 35.97 & 2.06 & 913 & 925 \\
\hline $\begin{array}{l}\text { 5-(4-methylphenoxy)-6-(4-nitrophenyl)- } \\
\text { 4-phenyl-1H-pyrimidin-2-one }\end{array}$ & 38.70 & 0.42 & 834 & 819 \\
\hline Squalene & 39.12 & 0.47 & 799 & 805 \\
\hline
\end{tabular}

aRT, Retention Time (min.).

bSI, Standard Index.

cRSI, Reverse Standard index.

Cyclohexylpentyl oxalate (8.7\%), 1,2-bis(TMS-oxy)-cyclooctene (8.6\%), techtoquinone (8.5\%), 3-(1,3-benzodioxol-5-yl)-5-hydroxy-4-nitrocyclohexanone $(7.2 \%)$, and 2-thiobarbituric acid, TMS (6.6\%) were the main compounds in Tectona grandis wood acetone extract (Table 8). The main compounds isolated from water extract of $T$. grandis were $(E)-4,4$-dimethyl-2-pentene $(51.14 \%)$, vinylather $(15.7 \%)$, and divinyl carbinol (7.6\%) (Table 9). 
Table 9. Chemical Constituents of $T$. grandis Wood Water Extract

\begin{tabular}{|c|c|c|c|c|}
\hline Compound Name & $\mathrm{RT}^{\mathrm{a}}$ & $\mathrm{Area}(\%)$ & $\mathrm{SI}^{\mathrm{b}}$ & $\mathrm{RSI}^{\mathrm{c}}$ \\
\hline 2-Methyl-2-(1-methylethyl)-oxirane & 3.61 & 2.38 & 741 & 742 \\
\hline 3-Heptene & 4.36 & 0.97 & 730 & 761 \\
\hline (E)-3-Heptene & 4.45 & 0.81 & 776 & 778 \\
\hline Hexane & 5.49 & 0.28 & 741 & 743 \\
\hline Vinylather & 5.84 & 15.73 & 740 & 742 \\
\hline 2,2-Dimethyl-3-propyloxirane & 6.29 & 2.20 & 789 & 796 \\
\hline 2-Methyl-hexane & 6.49 & 5.11 & 788 & 796 \\
\hline (E)-4,4-Dimethyl-2-pentene & 6.78 & 51.14 & 748 & 748 \\
\hline Divinyl carbinol & 7.36 & 7.63 & 727 & 727 \\
\hline Morpholine, 4- $\alpha$-D-Mannopyranose, 5TMS derivative & 22.34 & 0.27 & 762 & 801 \\
\hline Vanillic acid, 2TMS derivative & 24.13 & 0.42 & 757 & 797 \\
\hline Cyanuric acid, 3TMS derivative & 26.22 & 2.08 & 752 & 793 \\
\hline L-Rhamnose, 4TMS derivative & 26.75 & 0.27 & 904 & 937 \\
\hline (E)-3-Stilbenol & 30.64 & 2.18 & 901 & 908 \\
\hline 1-Methyl-2-pentamethyldisilanyloxycyclohexane & 35.36 & 5.92 & 899 & 907 \\
\hline 1-Ethyl-2-pentamethyldisilanyloxycyclohexane & 35.97 & 2.26 & 881 & 889 \\
\hline S-(+)-Reticuline & 36.45 & 0.26 & 812 & 824 \\
\hline 4-(3-chlorophenyl)-2-methylsulfonyl-thiophene-3- & 36.64 & 0.36 & 785 & 846 \\
\hline carbonitrile & & & & \\
\hline
\end{tabular}

aRT, Retention Time (min.).

bSI, Standard Index.

${ }^{\mathrm{c}} \mathrm{RSI}$, Reverse Standard index.

Table 10 presents the chemical compounds of Eucalyptus gomphocephala branch EO where the main compounds were $p$-cymene $(28.8 \%),(+)$ spathulenol $(13.0 \%), \Delta 3-$ carene (7.5\%), 2-methyl-3-phenylpropanal (3.9\%), and 1,8-cineole (3\%).

Table 11 presents the chemical constituents of $E$. paralias leaf extract where the main compounds were $1 \beta \mathrm{H}$-romneine $(26.3 \%), \beta$-amyrin, TMS derivative $(8.3 \%), 8$ bromo-neoisolongifolene $(10.0 \%), 3,5,6,7,8,8 \alpha$-hexahydro-4,8 $\alpha$-dimethyl-6-(1-methylethenyl)-2(1H)-naphthalenone $(5.7 \%)$ and 24 -methylene-cycloartenol, acetylated $(4.7 \%)$.

Table 10. Chemical Constituents of E. gomphocephala Branch Essential Oil

\begin{tabular}{|c|c|c|c|c|}
\hline Compound Name & $\mathrm{RT}^{\mathrm{a}}$ & Area (\%) & $\mathrm{Sl}^{\mathrm{b}}$ & $\mathrm{RSI}^{\mathrm{c}}$ \\
\hline$\Delta 3$-Carene & 7.34 & 7.46 & 781 & 785 \\
\hline Sabinene & 7.98 & 0.98 & 807 & 811 \\
\hline$\beta$-Pinene & 8.09 & 0.61 & 796 & 807 \\
\hline$\beta$-Myrcene & 8.23 & 0.47 & 786 & 835 \\
\hline L-Phellandrene & 8.54 & 0.56 & 793 & 797 \\
\hline$\alpha$-Terpinolene & 8.72 & 0.15 & 781 & 807 \\
\hline$p$-Cymene & 8.89 & 28.82 & 780 & 789 \\
\hline $1,8-$-Cineole & 9.02 & 3 & 821 & 837 \\
\hline cis-Linalool oxide & 9.59 & 0.26 & 807 & 811 \\
\hline trans-Linaloloxide & 9.84 & 0.25 & 802 & 815 \\
\hline Linalool & 10.04 & 1.25 & 787 & 798 \\
\hline trans-Caryophyllene & 10.35 & 0.17 & 832 & 835 \\
\hline 2-Cyclohexen-1-ol & 10.48 & 1.61 & 823 & 839 \\
\hline trans-Pinene hydrate & 10.78 & 1.87 & 806 & 810 \\
\hline Pinocarvone & 11.06 & 0.26 & 794 & 806 \\
\hline Cryptone & 11.55 & 17 & 814 & 832 \\
\hline
\end{tabular}




\begin{tabular}{|c|c|c|c|c|}
\hline cis-Carveol & 11.90 & 0.56 & 804 & 809 \\
\hline 2-Methyl-3-phenylpropanal & 12.28 & 3.91 & 799 & 812 \\
\hline Cyclohexadienemethanol & 12.67 & 0.57 & 779 & 808 \\
\hline Phellandral & 12.78 & 2.06 & 819 & 839 \\
\hline Cuminol & 12.94 & 1 & 805 & 812 \\
\hline Carvacrol & 13.02 & 2.97 & 802 & 817 \\
\hline Orivone & 13.21 & 0.48 & 786 & 816 \\
\hline trans-Caryophyllene & 15.12 & 0.32 & 824 & 845 \\
\hline Diosphenol I & 15.57 & 0.16 & 813 & 819 \\
\hline Aromadendrene 2 & 15.88 & 0.2 & 806 & 821 \\
\hline (-)-Caryophyllene oxide & 17.42 & 0.43 & 822 & 842 \\
\hline (+) Spathulenol & 18.01 & 13.05 & 816 & 822 \\
\hline Humulene oxide & 18.37 & 0.98 & 789 & 843 \\
\hline Isospathulenol & 18.65 & 0.44 & 789 & 843 \\
\hline Ledene & 19.23 & 0.11 & 820 & 841 \\
\hline $3,5,14,19-$ Card-20(22)-enolide & 19.86 & 0.17 & 814 & 821 \\
\hline -Cadinen-15-al & 20.00 & 0.19 & 804 & 820 \\
\hline
\end{tabular}

aRT, Retention Time (min.).

bSI, Standard Index.

'RSI, Reverse Standard index.

Table 11. Chemical Constituents of E. paralias Leaf Extract

\begin{tabular}{|c|c|c|c|c|}
\hline Compound Name & $\mathrm{RT}^{\mathrm{a}}$ & $\begin{array}{c}\text { Area } \\
(\%)\end{array}$ & $S I^{b}$ & $\mathrm{RSI}^{\mathrm{C}}$ \\
\hline 2,5-dimethyl-1,5-Heptadiene-3,4-diol & 5.84 & 0.63 & 789 & 843 \\
\hline$n$-Decanol tetrahydropyran ether & 6.49 & 0.25 & 826 & 850 \\
\hline (E)-4,4-Dimethyl-2-pentene & 6.78 & 2.18 & 810 & 817 \\
\hline N-Methylpyrrolin & 7.87 & 0.75 & 786 & 822 \\
\hline Methyl cis-3-chloropropenoate & 7.95 & 0.77 & 777 & 812 \\
\hline Vinylcyclohexyl ether & 8.97 & 0.32 & 828 & 850 \\
\hline Glycerol, TMS derivative & 14.34 & 0.51 & 808 & 814 \\
\hline Erythritol (4TMS) derivative & 19.59 & 0.20 & 795 & 803 \\
\hline$\beta$-Arabinose, TMS derivative & 22.31 & 0.19 & 786 & 820 \\
\hline Shikimic acid, 4TMS derivative & 25.14 & 1.03 & 824 & 846 \\
\hline 1,2,4,5-Cyclohexanetetrol, 4TMS derivative & 25.24 & 0.89 & 803 & 810 \\
\hline Tagatose, TMS derivative & 25.38 & 0.84 & 783 & 818 \\
\hline Myristic acid, TMS derivative & 25.45 & 0.33 & 767 & 802 \\
\hline Methyl- $\alpha$-D-ribofuranoside, 3TMS derivative & 25.87 & 0.20 & 820 & 841 \\
\hline$\alpha$-D-Glucopyranose, 5-TMS & 26.71 & 2.98 & 809 & 809 \\
\hline Gallic acid, 4TMS & 27.58 & 3.27 & 788 & 801 \\
\hline Palmitic acid, TMS & 28.65 & 0.96 & 824 & 846 \\
\hline Oleic Acid, (Z)-, TMS derivative & 31.24 & 0.17 & 799 & 806 \\
\hline Eicosane & 37.53 & 0.20 & 779 & 815 \\
\hline Dihydropseudoionone & 40.48 & 0.38 & 817 & 818 \\
\hline 9,9,10-Trimethyl-9,10-dihydroanthracene & 44.01 & 0.18 & 792 & 806 \\
\hline $\begin{array}{l}\text { 4H-1-Benzopyran-4-one, 8- } \beta \text {-D-glucopyranosyl-5,7-dimethoxy-2-(4- } \\
\text { methoxyphenyl)- }\end{array}$ & 44.54 & 0.29 & 845 & 862 \\
\hline Lanosterol, TMS derivative & 45.45 & 1.37 & 778 & 789 \\
\hline$\beta$-Sitosterol, TMS derivative & 45.55 & 0.48 & 755 & 759 \\
\hline $\begin{array}{c}\text { 3,5,6,7,8,8 } \alpha \text {-Hexahydro-4,8 } \text {-dimethyl-6-(1-methylethenyl)-2(1H)- } \\
\text { naphthalenone }\end{array}$ & 45.79 & 5.66 & 740 & 750 \\
\hline$\beta$-Amyrin, TMS derivative & 46.37 & 8.34 & 819 & 827 \\
\hline
\end{tabular}




\begin{tabular}{|c|c|c|c|c|}
\hline $1 \beta \mathrm{H}-$ Romneine & 46.53 & 26.28 & 793 & 825 \\
\hline Moretenol & 47.29 & 2.59 & 793 & 823 \\
\hline 24-methylene-cycloartenol, acetylated & 47.62 & 4.73 & 808 & 832 \\
\hline 7-allyloxy-4-methylcoumarin & 48.40 & 0.87 & 796 & 813 \\
\hline 8-bromo-Neoisolongifolene & 49.83 & 10.05 & 810 & 836 \\
\hline 5-Isopropylidene-6-methyldeca-3,6,9-trien-2-one & 50.14 & 1.43 & 800 & 808 \\
\hline 3-Hydroxy-4-phenyl-5-isopropyl-1,2,4-triazole & 50.84 & 2.27 & 793 & 811 \\
\hline
\end{tabular}

aRT, Retention Time (min.).

bSI, Standard Index.

cRSI, Reverse Standard index.

In E. paralias seed extract (Table 12) the main compounds were $\alpha$-linolenic acid, TMS (15.2\%), 2-(3-cyano-4,6-dimethyl-2-oxopyridin-1(2H)-yl)- $N$-(4-fluorophenyl) acetamide $(8.3 \%)$, 8-bromo-neoisolongifolene (7.5\%), gallic acid, 4TMS (5.7\%), $\alpha$-Dglucopyranose, 5TMS derivative (5.4\%), 24-methylene-cycloartenol, acetylated (5.3\%), palmitic acid, TMS (4.0\%), cyclohexylpentyl oxalate (3.3\%), shikimic acid (4TMS) $(3.2 \%)$ and $\beta$-amyrin-TMS (3.1\%).

Table 12. Chemical Constituents of E. paralias Seeds Extract

\begin{tabular}{|c|c|c|c|c|}
\hline Compound Name & $\begin{array}{l}\mathrm{RT}^{\mathrm{a}} \\
\text { (min.) }\end{array}$ & $\begin{array}{c}\text { Area } \\
(\%)\end{array}$ & $S I^{b}$ & $\mathrm{RSI}^{\mathrm{c}}$ \\
\hline Allyl $n$-octyl ether & 3.61 & 0.47 & 775 & 813 \\
\hline Vinylather & 5.85 & 1.08 & 812 & 836 \\
\hline$n$-Decanol tetrahydropyran ether & 6.49 & 0.35 & 800 & 817 \\
\hline Cyclohexylpentyl oxalate & 6.78 & 3.32 & 798 & 806 \\
\hline Glycerol, TMS derivative & 14.34 & 0.94 & 778 & 815 \\
\hline Theitol, 4TMS & 19.43 & 0.23 & 838 & 845 \\
\hline Erythritol (4TMS) & 19.58 & 0.81 & 810 & 837 \\
\hline 1-Deoxypentitol, 4TMS derivative & 20.24 & 0.22 & 806 & 815 \\
\hline D-Arabinose, 4TMS & 22.31 & 0.34 & 805 & 814 \\
\hline L-Fucitol TMS & 24.55 & 0.29 & 811 & 821 \\
\hline Shikimic acid (4TMS) & 25.14 & 3.24 & 808 & 836 \\
\hline 1,2,4,5-Cyclohexanetetrol, 4TMS derivative & 25.24 & 2.30 & 792 & 812 \\
\hline Tagatose, TMS derivative & 25.38 & 2.93 & 792 & 811 \\
\hline $\begin{array}{l}\text { L-Sorbopyranose, (1S,2R,3S)-, 5TMS } \\
\text { derivative }\end{array}$ & 25.46 & 0.80 & 810 & 841 \\
\hline Mannonic acid, 1,5-lactone, TMS & 25.86 & 0.22 & 808 & 820 \\
\hline$\beta$-Gulofuranose, TMS & 25.92 & 0.40 & 800 & 823 \\
\hline$\alpha$-D-Glucopyranose, 5TMS derivative & 26.71 & 5.44 & 779 & 796 \\
\hline Gallic acid, 4TMS & 27.58 & 5.66 & 821 & 823 \\
\hline Myo-Inositol (6TMS) & 27.80 & 0.30 & 804 & 823 \\
\hline Palmitic acid, TMS & 28.65 & 4.05 & 797 & 809 \\
\hline Esculetin, 2TMS derivative & 29.66 & 0.45 & 840 & 847 \\
\hline Linoleic acid, TMS & 31.15 & 2.01 & 806 & 811 \\
\hline$\alpha$-Linolenic acid, TMS & 31.26 & 15.18 & 807 & 840 \\
\hline Stearic acid, TMS derivative & 31.59 & 0.74 & 798 & 812 \\
\hline Galactose 5TMS & 34.14 & 0.30 & 774 & 822 \\
\hline 2,3,4,5-Tetraphenylpyrrole & 36.36 & 0.38 & 767 & 786 \\
\hline Monoolein TMS & 38.42 & 2.12 & 810 & 840 \\
\hline Monostearin TMS & 38.70 & 0.41 & 809 & 843 \\
\hline
\end{tabular}




\begin{tabular}{|c|c|c|c|c|}
\hline Propargyl methacrylate & 40.48 & 0.77 & 796 & 811 \\
\hline 2-Ethylacridine & 40.81 & 0.29 & 792 & 808 \\
\hline Deoxyglucose, 4TMS derivative & 41.00 & 0.25 & 779 & 793 \\
\hline$N, N$-Dimethyl-4-nitroso-3-aniline TMS & 44.01 & 0.28 & 774 & 788 \\
\hline 2-(1-Adamantyl)ethyl phenylacetate & 44.85 & 1.43 & 809 & 845 \\
\hline $\begin{array}{c}(3 \beta, 5 \alpha) \text {-Cholesta-8,24-dien-3-ol, TMS } \\
\text { derivative }\end{array}$ & 45.45 & 2.15 & 787 & 804 \\
\hline$\beta$-Sitosterol TMS & 45.54 & 1.59 & 779 & 793 \\
\hline $\begin{array}{l}\text { 3,5,6,7,8,8 } \alpha \text {-Hexahydro-4,8 } \alpha \text {-dimethyl-6-(1- } \\
\text { methylethenyl)-2(1H)-naphthalenone }\end{array}$ & 45.78 & 2.19 & 795 & 811 \\
\hline$\beta$-Amyrin, TMS derivative & 46.36 & 3.11 & 779 & 792 \\
\hline $\begin{array}{l}\text { 2-(3-cyano-4,6-dimethyl-2-oxopyridin-1(2H)- } \\
\text { yl)- } N \text {-(4-fluorophenyl)acetamide }\end{array}$ & 46.49 & 8.28 & 793 & 823 \\
\hline Moretenol & 47.28 & 2.65 & 788 & 806 \\
\hline 24-methylene-cycloartenol, acetylated & 47.62 & 5.34 & 784 & 797 \\
\hline 9-tert-Butyl-10-ethyl-9,10-dihydroanthracene & 49.45 & 1.65 & 768 & 770 \\
\hline 8-bromo-Neoisolongifolene & 49.81 & 7.51 & 815 & 835 \\
\hline 8S,14-Cedran-diol & 50.12 & 1.28 & 808 & 813 \\
\hline
\end{tabular}

aRT, Retention Time (min.).

bSI, Standard Index.

${ }^{\mathrm{c}} \mathrm{RSI}$, Reverse Standard index.

Table 13 shows chemical composition of peels of $P$. granatum extract: furfural $(32.1 \%)$, orotyl amide $(11.0 \%)$, D-allose $(9.2 \%)$, $n$-capric acid $(6.4 \%), 3,5$-dihydroxy-6methyl-2,3-dihydro-4H-pyran-4-one (6.1\%), 2,5-dimethylfurane (4.6\%), ethyl $\alpha$-D-glucopyranoside (4.3\%), cis-isoeugenol (3.9\%), and 1,6-anhydro- $\beta$-D-glucofuranose $(3.7 \%)$.

Table 13. Chemical Constituents of $P$. granatum Extract

\begin{tabular}{|c|c|c|c|c|}
\hline Compound name & $\mathrm{RT}^{\mathrm{a}}$ & Area $\%$ & $\mathrm{Sl}^{\mathrm{b}}$ & $\mathrm{RSI}^{\mathrm{c}}$ \\
\hline 2,5-Dimethylfurane & 5.59 & 4.56 & 803 & 818 \\
\hline Orotyl amide & 7.41 & 10.96 & 787 & 799 \\
\hline 2-Nonanol & 8.43 & 3.32 & 777 & 801 \\
\hline 2,3-Dihydroxy-propanal & 9.14 & 1.94 & 775 & 803 \\
\hline Bicyclo[2.2.1]heptane & 9.74 & 2.44 & 797 & 810 \\
\hline $\begin{array}{c}\text { 3,5-Dihydroxy-6-methyl-2,3-dihydro-4H-pyran-4- } \\
\text { one }\end{array}$ & 10.75 & 6.07 & 790 & 837 \\
\hline Heptanal & 11.54 & 0.77 & 775 & 829 \\
\hline Furfural & 11.96 & 32.09 & 770 & 820 \\
\hline cis-Isoeugenol & 13.78 & 3.87 & 762 & 885 \\
\hline Larixinic acid & 14.34 & 1.26 & 810 & 830 \\
\hline Semiphorone & 14.72 & 0.7 & 765 & 890 \\
\hline Cytidine & 15.12 & 0.31 & 755 & 770 \\
\hline D-Allose & 15.45 & 1.48 & 749 & 776 \\
\hline (+)-Endo-6-methyl-2-methylene- & 16.36 & 9.15 & 824 & 835 \\
\hline E-Anhydro- $\beta$-d-glucofuranose & 18.01 & 3.73 & 784 & 899 \\
\hline ch-D-glucopyranoside & 18.38 & 4.34 & 783 & 805 \\
\hline$n$-Capric acid & 19.37 & 1.18 & 783 & 795 \\
\hline Palmitic acid & 22.44 & 6.42 & 791 & 821 \\
\hline (mish.). & 22.73 & 1.32 & 786 & 806 \\
\hline
\end{tabular}

aRT, Retention Time (min.).

bSI, Standard Index.

'RSI, Reverse Standard index 
Table 14 presents the chemical composition of $O$. basilicum essential oil where the main compounds were estragole (65.9\%), eucalyptol (5.1\%), linalool (4.9\%), trans-4methoxycinnamaldehyde $(3.8 \%)$, and fenchyl acetate $(2.2 \%)$.

Table 14. Chemical Constituents of $O$. basilicum Essential Oil

\begin{tabular}{|c|c|c|c|c|}
\hline Name & $\mathrm{RT}^{\mathrm{a}}$ & Area $\%$ & $\mathrm{Sl}^{\mathrm{b}}$ & $\mathrm{RSI}^{\mathrm{c}}$ \\
\hline$\alpha$-Pinene & 6.01 & 0.84 & 782 & 817 \\
\hline$\beta$-Myrcene & 7.82 & 0.82 & 780 & 826 \\
\hline Eucalyptol & 9.08 & 5.10 & 802 & 838 \\
\hline 3-Carene & 9.73 & 0.34 & 772 & 798 \\
\hline L-Fenchone & 11.04 & 2.46 & 791 & 826 \\
\hline Linalool & 11.55 & 4.90 & 784 & 808 \\
\hline (+)-Fenchol & 11.93 & 0.24 & 775 & 800 \\
\hline Octen-1-ol acetate & 12.02 & 0.41 & 763 & 852 \\
\hline DL-Camphor & 13.01 & 2.93 & 796 & 813 \\
\hline$(-)-$ Terpinen-4-ol & 14.23 & 0.21 & 795 & 823 \\
\hline Estragole & 15.29 & 65.93 & 792 & 820 \\
\hline Acetic acid, octyl ester & 15.63 & 0.43 & 792 & 815 \\
\hline Fenchyl acetate & 15.88 & 2.23 & 795 & 810 \\
\hline$p$-Anisaldehyde & 16.92 & 1.64 & 775 & 812 \\
\hline Bornyl acetate & 18.07 & 1.13 & 772 & 815 \\
\hline$p$-Propyl anisole & 18.50 & 0.18 & 807 & 818 \\
\hline trans-Benzylideneacetone & 18.65 & 0.26 & 781 & 813 \\
\hline exo-2-Hydroxycineole acetate & 19.98 & 0.22 & 778 & 802 \\
\hline Methyl eugenol & 22.11 & 0.69 & 781 & 815 \\
\hline$\alpha$-Bergamotene & 23.11 & 2.11 & 781 & 804 \\
\hline trans- $\beta$-Farnesene & 24.63 & 0.23 & 860 & 883 \\
\hline$\gamma$-Muurolene & 25.52 & 0.55 & 855 & 884 \\
\hline -Mans-4-Moxycinnamaldehyde & 27.16 & 3.81 & 868 & 888 \\
\hline Spathulenol & 27.41 & 0.30 & 853 & 876 \\
\hline Carotol & 28.49 & 0.40 & 826 & 846 \\
\hline tau.-Cadinol & 29.25 & 2.02 & 833 & 853 \\
\hline trans & & & &
\end{tabular}

aRT, Retention Time (min.).

bSI, Standard Index.

'RSI, Reverse Standard index

The results showed that the EOs extracted in acetone of $T$. grandis, S. aromaticum, and E. gomphocephala have remarkable antifungal activities against the three tested pathogenic fungi, $A$. solani, $F$. oxysporum, and $R$. solani. The acetone extract of $T$. grandis showed the highest antifungal activity against $R$. solani and $A$. solani with $\mathrm{EC}_{50}$ values of 118 and $294 \mu \mathrm{g} / \mathrm{mL}$, respectively. However, T. grandis acetone extract showed lower antifungal activity against $F$. oxysporum. The other plant extracts from $S$. aromaticum and E. gomphocephala exhibited moderate antifungal effects against the three tested fungi. These results confirmed the antifungal activity of different plant extracts against three plant pathogenic fungi.

The major compounds (eugenol acetate, eugenol and caryophyllene (Nassar et al. 2007 ) of the EOs from $S$. aromaticum have been known to possess various antibacterial and antifungal properties ( Fu et al. 2007; Singh 2018). Eugenol, eugenol acetate, 
caryophellene, acetyle, eugenol, sesquiterpene ester, phenyl propanoid were the main compounds in the ethanolic extract from dried flower buds of S. aromaticum (Ghelardini et al. 2001; Miyazawa and Hisama 2003; Sumalatha et al. 2010). A 20\% concentration of flower bud aqueous extract of S. aromaticum showed 100\% inhibition of mycelial growth of Aspergillus niger (Avasthi et al. 2010). The antifungal effect of S. aromaticum was found on Aspergillus spp. and Penicillium spp. (Garg and Siddiqui 1992; Vazquez et al. 2001).

There was an increase in mycelial growth over time except for $50 \mu \mathrm{L} / 20 \mathrm{~mL}$ of PDA, where no mycelial growth was detected. In addition, the present results agree with other studies, where essential oils of $S$. aromaticum were used against various common fungal pathogens of plants namely, F. moniliforme, F. oxysporum, Aspergillus sp., Mucor sp., Trichophyton rubrum and Microsporum gypseum (Pinto et al. 2009; Rana et al. 2011; Sharma et al. 2016).

Extracts from almost every part of $T$. grandis were composed of chemical compounds from different classes such as flavonoids, steroidal compounds, glycosides, quinones, and phenolic acids (Ohmura et al. 2000), with remarkable antifungal and antitermitic effects (Healey and Gara 2003; Thulasidas and Bhat 2007; Shalini and Rachana 2009; Florence et al. 2012; Guerrero-Vásquez et al. 2013). Lapachol is a naphthoquinone and lapachonone, found in T. grandis wood and bark (Goel et al. 1987; Sumthong et al. 2006). Bis (2-ethylhexyl) phthalate has been isolated from wood extracts of $T$. grandis and is a good repellent to termites (Alabi and Oyeku 2007). Heartwood extract from T. grandis reduces the weight loss caused by white rots (Pleurotus squarrosulus and Lentinus subnudus) in two hardwood species (Triplochiton scleroxylon and Gmelina arborea) (Adegeye et al. 2009).

Naphtoquinone, anthraquinone (Thulasidas and Bhat 2007), and 2-methyl anthraquinone (techtoquinone) (Windeisen et al. 2003) that inhibited the growth of of Coniophora puteana, a brown rot fungus (Haupt et al., 2003). Lapachol and deoxylapachol are naphtoquinone derivatives which also reported as biologically active compound (Windeisen et al. 2003). Plant extracts containing naphtoquinones (chromatic pigments) have been used for cancer and rheumatoid arthritis treatment (Babula et al. 2006). Lapachol is a natural quinone has been isolated from heartwood of Asian and South American Bignoniaceae (Tabebuia, Taigu, and Tecoma) (Steinert et al. 1995).

The sawdust of $T$. grandis contains the active components deoxylapachol and tectoquinnone, which inhibited the growth of A. niger (Neamatallah et al. 2005; Sumthong et al. 2006; Hussain et al. 2007). Furthermore, its leaf extract significantly suppressed the growth of Arthrinium phaeospermum (Astiti and Suprapta 2012). Leaf and bark extracts of T. grandis also have antifungal activity against $A$. niger, Trichoderma viride, and $A$. flavus (Lanka and Parimala 2017).

Previous studies have shown that $n$-hentriacontane, $n$-nonacosane, $n$-triacontane, $n$ dotriacontane, $n$-tritriacontane, and $n$-pentatriacontane, hexacosanol, octacosanol, cycloartenol, methylenecycloartenol, $\beta$-sitosterol, stigmasterol, campesterol, cholesterol, oleanolic, betulin and $\beta$-amyrin were isolated from extracts of E. paralias (Rizk et al. 1974; Shi et al. 2008; Noori et al. 2009) and have potential antimicrobial activities (Jassbi 2006; Shi et al. 2008; Noori et al. 2009).

Many previous studies reported that the EO of Eucalyptus sp. completely inhibited mycelial growth of plant pathogens. $p$-cymene (17.2\%) and crypton (8.9\%) were the main compounds in E. gomphocephala leaf essential oil (Salem et al. 2015). The EOs of extracts from different parts of E. gomphocephala have been reported to have potential 
antimicrobial activities (Salem et al. 2015; Elansary et al. 2017). EOs of Eucalyptus sp. have been shown to have antibacterial and antifungal activities (Bendaoud et al. 2009; Bachheti et al. 2011). The antimicrobial/antifungal activities of essential oils are generally due to the presence of components such as 1,8-cineole, citronellal, citronellol, citronellyl acetate, $p$-cymene, eucamalol, limonene, and linalool (Nezhad et al. 2009).

Alternaria alternata, Stemphylium botryosum, and Fusarium spp. were significantly inhibited by the aqueous extracts of pomegranate peels (Glazer et al. 2012). Methanolic extracts of pomegranate showed inhibitory activity against A. niger, Penicillium citrinum, Rhizopus oryzae, and Trichoderma reesei (Dahham et al. 2010).

Phenolic compounds such as punicalagin, punicalin, granatins $\mathrm{A}$ and $\mathrm{B}$, gallagyldilacton, tellimagrandin I, pedunculagin, and corilagin were isolated from peel extract of Punica granatum were responsible for the antimicrobial activity (Fetrow and Avila 2000; Catão et al. 2006; Dudonné et al. 2009). Tannins, flavonoids, and alkaloids presented in peel aqueous extract of $P$. granatum were observed positive tests against diarrhea (Qnais et al. 2007). P. granatum peel methanolic extract showed antifungal activity against Candidia albicans (Höfling et al. 2010). Alcoholic and hot water extracts of $P$. granatum peel with high amount of gallotanic acid showed good antifungal activities against $C$. albicans, $C$. tropicalis, $A$. fumegatus, and $A$. nigar (Shaokat et al. 2007). Many tactics have been developed to control the threat of mosquito-borne disease. One such tactic is to kill mosquito larvae, using a strategy based on synthetic insecticides. Although they are effective, they have generated many problems such as insecticide resistance, environmental pollution, and adverse effects on human beings and livestock (Liu et al. 2005; Lixin et al. 2006).

The present study revealed that the EO of $O$. basilicum has a potent larvicidal activity against $C$. pipiens larvae. In line with our results, the larvicidal activity of $O$. basilicum EO was reported against four other mosquito species Culex tritaeniorhynchus, Aedes albopictus, Anopheles subpictus, and Aedes aegypti with $\mathrm{LC}_{50}$ values of 14.01, 11.97, 9.75, and 75.35 ppm, respectively (Govindarajan et al. 2013; Manzoor et al. 2013). Furthermore, the EO of $O$. basilicum displayed the highest larvicidal activity against the larvae of the lymphatic filariasis vector (Culex quinquefasciatus), in comparison to $O$. sanctum and $O$. gratissimum (Rajamma et al. 2011). Diverse species of Ocimum from different countries displayed a potent larvicidal activity against mosquito larvae such as $O$. sanctum L. from India and Nigeria (Pathak et al. 2000; Gbolade and Lockwood 2008), O. americanum L. and $O$. gratissimum L. from Brazil (Cavalcanti et al. 2004), and $O$. lamiifolium Hochst./O. suave Willd from Ethiopia (Massebo et al. 2009). This activity could be related to the presence of important chemical compounds in the EOs. The main compounds found in the EO of O. basilicum were stragole and eucalyptol. Previously, the major EO constituents of $O$. basilicum plants included methyl chavicol (estragole), linalool, eugenol, and 1,8-cineole (eucalyptol) (Sajjadi 2006; Telci et al. 2006; Chalchat and Özcan 2008; Pripdeevech et al. 2010).

The Eucalyptus genus (Family: Mitraceae) includes more than 700 species and is one of the world's most important widely planted genera (Menut et al. 1995). In the present study, E. gomphocephala EO showed a remarkable larvicidal activity $\left(\mathrm{LC}_{50}=30.07 \mathrm{mg} / \mathrm{L}\right)$ against $C$. pipiens. Confirming the present results, similar larvicidal activity of $E$. camaldulensis $\mathrm{EO}$ was reported with an $\mathrm{LC}_{50}$ value of $31 \mu \mathrm{g} / \mathrm{mL}$ against $A$. aegypti (Cheng et al. 2009). S. aromaticum is an evergreen tree in the family Myrtaceae. Its aromatic flower buds are widely used in traditional medicine (Alqareer et al. 2012). The methanolic extract of $S$. aromaticum displayed higher larvicidal activity $\left(\mathrm{LC}_{50}=58.73 \mathrm{mg} / \mathrm{L}\right)$ against $C$. 
pipiens compared with its $\mathrm{EO}\left(\mathrm{LC}_{50}=128.92 \mathrm{mg} / \mathrm{L}\right)$. A very similar result of the larvicidal activity of the EO from $S$. aromaticum was reported against $A$. aegypti $\left(\mathrm{LC}_{50}=124.69\right.$ $\mathrm{ppm}$ ) and C. quinquefasciatus $\left(\mathrm{LC}_{50}=124.42 \mathrm{mg} / \mathrm{L}\right.$ ) (Sutthanont et al. 2010; Fayemiwo et al. 2014).

\section{CONCLUSIONS}

1. The essential oils extracted from T. grandis (acetone extract), S. aromaticum (methanolic extract), and E. gomphocephala (branch oil) had remarkable antifungal activities against the three tested pathogenic fungi, A. solani, F. oxysporum, and $R$. solani.

2. The essential oils from O. basilicum, E. gomphocephala, and S. aromaticum as well as the methanolic extract of $S$. aromaticum have remarkable larvicidal effects.

3. By GC/MS analysis, the most abundant compounds identified in S. aromaticum methanolic extract were 3-allylguaiacol and eugenol acetate; in S. aromaticum essential oil were eugenol acetate, isoeugenol, trans-caryophyllene and $\alpha$-humulene; in Tectona grandis wood acetone extract were cyclohexylpentyl oxalate, 1,2-bis(TMS-oxy)cyclooctene, techtoquinone, 3-(1,3-benzodioxol-5-yl)-5-hydroxy-4-nitrocyclohexanone, and 2-thiobarbituric acid, TMS; in T. grandis water extract were $(E)-4,4-$ dimethyl-2-pentene, vinylather, and divinyl carbinol; in E. gomphocephala branch oil were $p$-cymene, (+)spathulenol, $\Delta 3$-carene, 2-methyl-3-phenylpropanal, and 1,8cineole; in $E$. paralias leaf extract were $1 \beta \mathrm{H}$-romneine, $\beta$-amyrin, TMS derivative, and 8-bromo-neoisolongifolene; in E. paralias seed extract were $\alpha$-linolenic acid, TMS, 2 (3-cyano-4,6-dimethyl-2-oxopyridin-1(2H)-yl)- $N$-(4-fluorophenyl)acetamide, $\quad 8$ bromo-neoisolongifolene, gallic acid, 4TMS, $\alpha$-D-glucopyranose, 5TMS derivative, 24-methylene-cycloartenol, acetylated, palmitic acid, TMS, cyclohexyl pentyl oxalate, Shikimic acid (4TMS), and $\beta$-amyrin-TMS, and in $P$. granatum peel extract were furfural, orotyl amide, $D$-allose, $n$-capric acid, 3,5-dihydroxy-6-methyl-2,3-dihydro4H-pyran-4-one, 2,5-dimethylfurane, ethyl $\alpha$-D-glucopyranoside, and cis-isoeugenol; in $O$. basilicum essential oil were estragole, eucalyptol, linalool, trans-4methoxycinnamaldehyde and fenchyl acetate.

4. These results support the idea of using natural plant extracts or essential oils as alternative antifungal compounds to control phytopathogenic fungi and the mosquito population.

5. This application could reduce the use of synthetic fungicides and insecticides.

\section{ACKNOWLEDGMENTS}

The authors extend their appreciation to the Deanship of Scientific Research at King Saud University for funding this work through research group No. (RG- 1440-028). The authors thank the RSSU at King Saud University for their technical support. 


\section{REFERENCES CITED}

Abbott, W. S. (1925). "A method of computing the effectiveness of an insecticide," Journal of Economic Entomology 18(1), 265-267. DOI: 10.1093/jee/18.2.265a

Adegeye, A. O., Ogunsanwo, O. Y., and Olajuyigbe, S. O. (2009). "Antifungal activities of heart wood extract (HWE) of teak Tectona grandis against two white rots in woods of Gmelina arborea and Triplochiton scleroxylon," Academic Journal of Plant Sciences 2 (4), 279-285.

Avasthi, S., Gautam, A. K., and Bhadauria, R. (2010). “Antifungal activity of plant products against Aspergillus niger: A potential application in the control of a spoilage fungus," Biological Forum - An International Journal 2(1), 53-55.

Alabi, K., and Oyeku, T. (2007). "The chemical constituents extractable from teak tree (Tectona grandis Linn) obtained from Fountain University, Osogbo," Nigerian Journal of Basic and Applied Science 25(1), 73-80. DOI: 10.4314/njbas.v25i1.10

Alqareer, A., Alyahya, A., and Andersson, L. (2012). "The effect of clove and benzocaine versus placebo as topical anesthetics," Journal of Dentistry 34(10), 74750. DOI: 10.1016/j.jdent.2006.01.009

Amadi, J. E., Salami, S. O., and Eze, C. S. (2010). “Antifungal properties and phytochemical screening of extracts of African basil (Ocimum gratissimum L.)," Agriculture and Biology Journal of North America ISSN Print: 2151-7517.

Astiti, N. P. A., and Suprapta, D. N. (2012). "Antifungal activity of teak (Tectona grandis L.F) against Arthrinium phaeospermum (Corda) M.B. Ellis, the cause of wood decay on Albizia falcataria (L.) Fosberg," Journal of the International Society for Southeast Asian Agricultural Sciences 18(1), 62-69.

Babula, P., Mikelova, R., Adam, V., Kizek, R., Havel, L. and Sladky, Z. (2006). "Naphthoquinones-biosynthesis, occurrence and metabolism in plants," Ceska a Slovenskáfarmacie 55(4), 151-159.

Bachheti, R. K., Joshi, A., and Singh, A. (2011). "Oil content variation and antimicrobial activity of Eucalyptus leaves oils of three different species of Dehradun, Uttarakhand, India," International Journal of ChemTech Research 3(2), 625-628.

Badawy, M. E. I., and Abdelgaleil, S. A. M. (2014). "Composition and antimicrobial activity of essential oils isolated from Egyptian plants against plant pathogenic bacteria and fungi," Industrial Crops and Products 52(1), 776-782. DOI: 10.1016/j.indcrop.2013.12.003

Bendaoud, H., Bouajila, J., Rhouma, A., Savagnac, A., and Romdhane, M. (2009). "GC/MS analysis and antimicrobial and antioxidant activities of essential oil of Eucalyptus radiata," Journal of the Science of Food and Agriculture 89(8), 1292 1297. DOI: 10.1002/jsfa.3585

Catão, R. M. R., Antunes, R. M. O., Arruda, T. A., Pereira, M. S. V., Higino, J. S., Alves, J. A., Passos, M. G. V. M., and Santos, V.L. (2006). "Antimicrobial activity 'in vitro' of the ethanol extract Punica granatum against of Staphylococcus," Revista Brasileira de Análises Clínicas 38(2), 111-114.

Cavalcanti, E. S. B., Morais, S. M., Lima, M. A. A., and Santana, E. W. P. (2004). "Larvicidal activity of essential oils from Brazilian plants against Aedes aegypti L.," Memorias do Instituto Oswaldo Cruz 99(5), 541-544. DOI: 10.1590/S007402762004000500015 
Chalchat, J.-C., and Özcan, M. M. (2008). "Comparative essential oil composition of flowers, leaves and stems of basil (Ocimum basilicum L.) used as herb," Food Chemistry 110(2), 501-503. DOI: 10.1016/j.foodchem.2008.02.018

Cheng, S., Huang, C., Chen, Y., Yu, J., Chen, W., and Chang, S. (2009). "Chemical compositions and larvicidal activities of leaf essential oils from two eucalyptus species," Bioresource Technology 100(1), 452-456. DOI:

10.1016/j.biortech.2008.02.038

Dahham, S. S., Ali, M. N., Tabassum, H., and Khan, M. (2010). "Studies on antibacterial and antifungal activity of pomegranate (Punica granatum L.)," American-Eurasian Journal of Agricultural and Environmental Sciences 9(3), 273-281.

Dudonné, S., Vitrac, X., Coutière, P., Woillez, M., and Mérillon, J. M. (2009).

"Comparative study of antioxidant properties and total phenolic content of 30 plant extracts of industrial interest using DPPH, ABTS, FRAP, SOD, and ORAC Assays," Journal of Agricultural and Food Chemistry 57 (5), 1768-1774. DOI: 10.1021/jf803011r

Elansary, H. O., Salem, M. Z. M., Ashmawy, N. A., Yessoufou, K., and El-Settawy, A. A. (2017). "In vitro antibacterial, antifungal, and antioxidant activities of Eucalyptus spp. leaf extracts related to phenolic composition," Natural Product Research 31(24), 2927-2930. DOI: 10.1080/14786419.2017.1303698

Fayemiwo, K. A., Adeleke, M. A., Okoro, O. P., Awojide, S. H., and Awoniyi, O. I. (2014). "Larvicidal efficacies and chemical composition of essential oils of Pinus sylvestris and Syzygium aromaticum against mosquitoes," Asian Pacific Journal of Tropical Biomedicine 4(1), 30-34. DOI: 10.1016/S2221-1691(14)60204-5

Fetrow, C. W., and Avila, J. R. (2000). "Manual de Medicina Alternativa para o Profissional," Guanabara Koogan, Rio de Janeiro, pp 590-592.

Finney, D. J. (1971). Probit Analysis ( $3^{\text {rd }}$ Ed.), Cambridge University Press, London, UK. Florence, B. N., Nadine, A., Didier, S., Gilles, C., Yves, L., and Adjumane, A. K. (2012). “4',5'-Dihydroxy-epiisocatalponol, a new naphthoquinone from Tectona grandis L. $\mathrm{f}$. heartwood, and fungicidal activity," International Biodeterioration and Biodegradation 74, 93-98. DOI: 10.1016/j.ibiod.2012.03.010

Fu, Y., Zu, Y., Chen, L., Shi, X., Wang, Z., Sun, S., and Efferth, T. (2007). "Antimicrobial activity of clove and rosemary essential oils alone and in combination," Phytotherapy Research 21(10), 989-994. DOI: 10.1002/ptr.2179

Gakuubi, M. M., Maina, A. W., and Wagacha, J. M. (2017). "Antifungal activity of essential oil of Eucalyptus camaldulensis Dehnh. against selected Fusarium spp.," International Journal of Microbiology 2017, 1-7. DOI: 10.1155/2017/8761610

Garg, S. C., and Siddiqui, N. (1992). "Antifungal activity of some essential oil isolates," Pharmazie 47(6), 467-468.

Gbolade, A. A., and Lockwood, G. B. (2008). "Toxicity of Ocimum sanctum L. essential oil to Aedes aegypti larvae and its chemical composition," Journal of Essential OilBearing Plants 11(2), 148-153. DOI: 10.1080/0972060X.2008.10643611

Ghelardini, C., Galeotti, N., Di Cesare Mannelli, L., Mazzanti, G., and Bartoloini, A. (2001). "Local anaesthetic activity of beta-caryophyllene," Farmaco 56(5-7), 387389. DOI: 10.1016/S0014-827X(01)01092-8

Glazer, I., Masaphy, S., Prosper Marciano, P., Bar-Ilan, I., Holland, D., Kerem Z., and Amir, R. (2012). "Partial identification of antifungal compounds from Punica granatum peel extracts," Journal of Agriculture and Food Chemistry 60(19), 48414848 DOI: 10.1021/jf300330y 
Goel, R. K., Pathak, N. K., Biswas, M., Pandey, V. B., and Sanyal, A. K. (1987). "Effect of lapachol, a naphthaquinone isolated from Tectona grandis, on experimental peptic ulcer and gastric secretion," Journal of Pharmacy and Pharmacology 39(2), 138-140. DOI: 10.1111/j.2042-7158.1987.tb06962.x

Govindarajan, M., Sivakumar, R., Rajeswary, M., and Yogalakshmi, K. (2013).

"Chemical composition and larvicidal activity of essential oil from Ocimum basilicum (L.) against Culex tritaeniorhynchus, Aedes albopictus and Anopheles subpictus (Diptera: Culicidae)," Experimental Parasitology 134(1), 7-11. DOI: 10.1016/j.exppara.2013.01.018

Guerrero-Vásquez, G. A., Carlos Kleber, Z. A., Jose, M. G., and Francisco, A. M. (2013). "Practical first total synthesis of the potent phytotoxic (+)-naphthotectone, isolated from Tectona grandis," European Journal of Organic Chemistry 2013(27), 61756180. DOI: $10.1002 /$ ejoc. 201300783

Hahn, M. (2014). "The rising threat of fungicide resistance in plant pathogenic fungi: Botrytis as a case study," Journal of Chemical Biology 7(4), 133-141. DOI: 10.1007/s12154-014-0113-1

Healey, S. P., and Gara, R. I. (2003). "The effect of a teak (Tectona grandis) plantation on the establishment of native species in an abandoned pasture in Costa Rica," Forest Ecology and Management 176(1-3), 497-507. DOI: 10.1016/S0378-1127(02)00235-9

Horbach, R., Navarro-Quesada, A. R., Knogge, W., and Deising, H. B. (2011). "When and how to kill a plant cell: Infection strategies of plant pathogenic fungi," Journal of Plant Physiology 168(1), 51- 62. DOI: 10.1016/j.jplph.2010.06.014

Höfling, J. F., Anibal, P. C., Obando-Pereda, G. A., Peixoto, I. A., Furletti, V. F., Foglio, M. A., and Gonçalves, R. B. (2010). "Antimicrobial potential of some plant extracts against Candida species," Brazilian Journal of Biology 70(4), 1065-1068. DOI: 10.1590/S1519-69842010000500022

Hussain, H., Krohn, K., Uddin-Ahmad, V., Abbas-Miana, G., and Greend, I. R. (2007). "Lapachol: An overview," ARKIVOC 2007(2), 145-71. DOI: 10.3998/ark.5550190.0008.204

Jassbi, A. R. (2006). "Chemistry and biological activity of secondary metabolites in Euphorbia from Iran," Phytochemistry 67(18), 1977-1984. DOI: 10.1016/j.phytochem.2006.06.030

Liu, H., Xu, Q., Zhang, L., and Liu, N. (2005). "Chlorpyrifos resistance in Mosquito Culex quinquefasciatus," Journal of Medical Entomology 42(5), 815-820. DOI: 10.1093/jmedent/42.5.815

Lixin, S., Huiquin, D., Chongxia, G., Jin, Q., Jing, S., Lei, M., and Chang, L. Z. (2006). "Larvicidal activity of extracts of Ginko biloba exocarp for three different strains of Culex pipiens pallens," Journal of Medical Entomology 43(2), 258-261. DOI: 10.1093/jmedent/43.2.258

Mahboubi, M., and Bidgoli, F. G. (2010). "In vitro synergistic efficacy of combination of amphotericin B with Myrtus communis essential oil against clinical isolates of Candida albicans," Phytomed. 17(10), 771-774. DOI: 10.1016/j.phymed.2010.01.016

Manzoor, F., Samreen, K. B., and Parveen, Z. (2013). "Larvicidal activity of essential oils against Aedes aegypti and Culex quinquefasciatus larvae (Diptera: Culicidae)," Journal of Animal and Plant Science 23(2), 420-424. 
Massebo, F., Tadesse, M., Bekele, T., Balkew, M., and Gebre-Michael, T. (2009). "Evaluation on larvicidal effects of essential oils of some local plants against Anopheles arabiensis Patton and Aedes aegypti Linnaeus (Diptera, Culicidae) in Ethiopia," African Journal of Biotechnol. 8(17), 4183-4188.

Miyazawa, M., and Hisama, M. (2003). "Antimutagenic activity of phenylpropanoids from clove. (Syzygium aromaticum)," Journal of Agriculture and Food Chemistry 51(22), 6413-5422. DOI: 10.1021/jf030247q

Nassar, M. I., Gaara, A. H., Ghorab, A. H., Farrag, A. R., Shen, H., Huq, E., and Mabry, T. J. (2007). "Chemical constituents of clove (Syzygium aromaticum, Fam. Myrtaceae) and their antioxidant activity," Rev Latinoameri de Quimica 35(3), 47-57.

Neamatallah, A., Yan, L., Dewar, S. J., and Austin, B. (2005). "An extract from teak (Tectona grandis) bark inhibited Listeria monocytogenes and methicillin resistant Staphylococcus aureus," Letters in Applied Microbiology 41(1), 94-96. DOI: 10.1111/j.1472-765X.2005.01680.x

Nezhad, F. M., Zeigham, H., Mota, A., Sattari, M., and Yadegar A. (2009). "Antibacterial activity of Eucalyptus extracts on methicillin resistance of Staphylococcus aureus," Research Journal of Biological Sciences 4(5), 905-908.

NIST/EPA/NIH Mass Spectral Library (NIST 11) and NIST Mass Spectral Search Program (Version 2.0g) May 2011.

Noori, M., Chehreghani, A., and Kaveh, M. (2009). "Flavonoids of 17 species of Euphorbia (Euphorbiaceae) in Iran," Toxicological and Environmental Chemistry 91(4), 631-641. DOI: 10.1080/02772240802389936.

Oberacher, H. (2011). “Wiley Registry of Tandem Mass Spectral Data,” MS for ID, John Wiley \& Sons Inc: Hoboken, 2011.

Odalo, J., Omolo, M., Malebo, H., Angira, J., Njeru, P., Ndiege, I., and Hassanali, A. (2005). "Repellency of essential oils of some plants from Kenyan coast against Anopheles gambiae," Acta Tropica 95(3), 210-218. DOI: 10.1016/j.actatropica.2005.06.007

Ohmura, W., Doi, S., Aoyama, M., and Ohara, S. (2000). "Antifeedant activity of flavonoids and related compounds against the subterrarean termites," Journal of Wood Science 46(2), 147-153. DOI: 10.1007/BF00777362

Pandey, D. K., Tripathi, N. N., Tripathi, R. D., and Dixit, S. N. (1982). "Fungitoxic and phytotoxic properties of the essential oil of Hyptis suaveolens," Journal of Plant Diseases and Protection 89(6), 344-349.

Pathak, N., Mittal, P. K., Singh, O. P., Sagar, D. V., and Vasudevan, P. (2000). "Larvicidal action of essential oils from plants against the vector mosquitoes Anopheles stephensi (Liston), Culex quinquefasciatus(Say) and Aedes aegypti (L.)," International Pest Control 42(2), 53-55.

Pinto, E., Vale-Silva, L., Cavaleiro, C., and Salgueiro, L. (2009). "Antifungal activity of the clove essential oil from Syzygium aromaticum on Candida, Aspergillus and dermatophyte species," Journal of Medical Microbiology 58(Pt 11), 1454-1462. DOI: 10.1099/jmm.0.010538-0

Pripdeevech, P., Chumpolsri, W., Suttiarporn, P., and Wongpornchai, S. (2010). "The chemical composition and antioxidant activities of basil from Thailand using retention indices and comprehensive two-dimensional gas chromatography," Journal of Serbian Chemical Society 75 (11), 1503-1513. DOI: 10.2298/JSC100203125P 
Qnais, E. Y., Elokda A. S., Abu Ghalyun, Y. Y., and Abdulla, F. A. (2007). "Antidiarrheal activity of the aqueous extract of Punica granatum (Pomegranate) peels," Pharmaceutical Biology 45(9), 715-720. DOI: 10.1080/13880200701575304

Rahimi-Nasrabadi, M., Nazarian, S., Farahani, H., Fallah Koohbijari, G. R., Ahmadi, F., and Batooli, H. (2013). "Chemical composition, antioxidant, and antibacterial activities of the essential oil and methanol extracts of Eucalyptus largiflorens $\mathrm{F}$. Muell," International Journal of Food Properties 16(2), 369-381. DOI: 10.1080/10942912.2010.551310

Rahimi-Nasrabadi, M., Pourmortazavi, S. M., Nazarian, S., Ahmadi, F., and Batooli, H. (2013). "Chemical composition, antioxidant, and antibacterial activities of the essential oil and methanol extracts of Eucalyptus oleosa leaves," International Journal of Food Properties 16(5), 1080-1091. DOI: 10.1080/10942912.2011.558227

Rahimi-Nasrabadi, M., Ahmadi, F., and Batooli, H. (2012). "Essential oil composition of Eucalyptus procera Dehnh. leaves from central Iran," Natural Product Research 26(7), 637-642. DOI: 10.1080/14786419.2010.541875

Rajamma, A. J., Dubey, S., Sateesha, S. B., Tiwari, S. N., and Ghosh, S. K. (2011). "Comparative larvicidal activity of different species of Ocimum against Culex quinquefasciatus," Natural Products Research 25(20), 1916-1922. DOI: 10.1080/14786419.2010.551755

Rana, I. S., Rana, A. S., and Rajak, R. C. (2011). "Evaluation of antifungal activity in essential oil of the Syzygium aromaticum (L.) by extraction, purification and analysis of its main component eugenol," Brazilian Journal of Microbiology 42(4), 12691277. DOI: 10.1590/S1517-83822011000400004

Rizk, A. M., Youssef, A. M., Diab, M. A., and Salem, H. M. (1974). "Constituents of Egyptian Euphorbiaceae, I: Triterpenoids and related substances of Euphorbia paralias," Zeitschrift für Naturforschung 29c(9-10), 529-531.

Sajjadi, S. E. (2006). "Analysis of the essential oils of two cultivated basil (Ocimum basilicum L.) from Iran,” DARU Journal of Pharmaceutical Sciences 14(3), 128-130.

Salem, M. Z. M., Ali, H. M., El-Shanhorey, N. A., and Abdel-Megeed A. (2013). "Evaluation of extracts and essential oil from Callistemon viminalis leaves: Antibacterial and antioxidant activities, total phenolic and flavonoid contents," Asian Pacific Journal of Tropical Medicine 6(10), 785-791. DOI: 10.1016/S19957645(13)60139-X

Salem, M. Z. M., Zidan, Y. E., Mansour, M. M. A., El Hadidi, N. M. N., and Abo Elgat, W. A. A. (2016). "Antifungal activities of two essential oils used in the treatment of three commercial woods deteriorated by five common mold fungi," International Biodeterioration \& Biodegradation 106, 88-96. DOI: 10.1016/j.ibiod.2015.10.010

Salem, M. Z. M., Zidan, Y. E., Mansour, M. M. A., El Hadidi, N. M. N., and Abo Elgat, W. A. A. (2016). "Evaluation of usage three natural extracts applied to three commercial wood species against five common molds," International Biodeterioration \& Biodegradation 110, 206-226. DOI: 10.1016/j.ibiod.2016.03.028.

Salem, M. Z. M., Mansour, M. M. A., and Elansary H. O. (2019). "Evaluation of the effect of inner and outer bark extracts of sugar maple (Acer saccharum var. saccharum) in combination with citric acid against the growth of three common molds," Journal of Wood Chemical Technology 39(2), 136-147. DOI: 10.1080/02773813.2018.1547763

Salem, M. Z. M., Ashmawy, N. A., Elansary, H. O., and El-Settawy, A. A. (2015). "Chemotyping of diverse Eucalyptus species grown in Egypt and antioxidant and 
antibacterial activities of its respective essential oils," Natural Product Research 29(7), 681-685. DOI: 10.1080/14786419.2014.981539

Sales, M. D. C., Costa, H. B., Fernandes, P. M. B., Ventura, J. A., and Meira, D. D. (2016). "Antifungal activity of plant extracts with potential to control plant pathogens in pineapple," Asian Pacific Journal of Tropical Biomedicine 6(1), 26-31. DOI: 10.1016/j.apjtb.2015.09.026

Singh, R. (2018). "Antimicrobial activity of selected medicinal plants cinnamon and clove," International Journal of Research in Pharmacy and Pharmaceutical Sciences 3(5), 48-50. DOI: 10.22271/pharmacy

Shalini, and Rachana, S. (2009). "Antifungal activity screening and HPLC analysis of crude extract from Tectona grandis, Shilajit, Valeriana Wallachi," Electronic Journal of Environmental, Agricultural, and Food Chemistry 8(4), 218-229.

Sharma, A., Rajendran, S., Srivastava, A., Sharma, S. and Kundu, B. (2016). “Antifungal activities of selected essential oils against Fusarium oxysporum f. sp. lycopersici 1322, with emphasis on Syzygium aromaticum essential oil," Journal of Bioscience and Bioengineering 123(3), 308-313. DOI: 10.1016/j.jbiosc.2016.09.011

Shaokat, S. S., Hameed, H. A., and Mohammad H. J. (2007). "Anti-fungal activity of Punica granatum L. peels powder and extracts from pathogenic samples, ” Iraqi J. Pharm. Sci. 16(2), 12-20.

Shi, Q. W., Su, X. H., and Kiyota, H. (2008). "Chemical and pharmacological research of the plants in genus Euphorbia," Chemistry Reviews 108(10), 4295-4327. DOI: $10.1021 / \mathrm{cr} 078350 \mathrm{~s}$

Sumalatha, K., Kumar, A. S., and Lakshmi, M. S. (2010). "Review on natural aphrodisiac potentials to treat sexual dysfunction," International Journal of Pharmacy \& Therapeutics 1(1), 6-14.

Sumthong, P., Damveld, R. A., Choi, Y. H., Arentshorst, M., Ram, A. F., van den Hondel, C. A., and Verpoorte, R. (2006). "Activity of quinines from teak (Tectona grandis) on fungal cell wall stress," Planta Medica 72(10), 943-944. DOI: 10.1055/s2006-946676

Sutthanont, N., Choochote, W., Tuetun, B., Junkum, A., Jitpakdi, A., Chaithong, U., Riyong, D., and Pitasawat, B. (2010). "Chemical composition and larvicidal activity of edible plant-derived essential oils against the pyrethroid-susceptible and -resistant strains of Aedes aegypti (Diptera: Culicidae)," Journal of Vector Ecology 35, 106115.

Steinert, J., Khalaf, H., and Rimpler, M. (1995). "HPLC separation and determination of naphtho[2,3-b]furan-4,9-diones and related compounds in extracts of Tabebuia avellanedae (Bignoniaceae)," Journal of Chromatography A 693(2), 281-287. https://doi.org/10.1016/0021-9673(94)01128-2

Telci, I., Bayram, E., Yılmaz, G., and Avc1, B. (2006). "Variability in essential oil composition of Turkish basils (Ocimum basilicum L.)," Biochemical Systematics and Ecology 34(6), 489-497. DOI: 10.1016/j.bse.2006.01.009

Thulasidas, P. K., and Bhat, K. M. (2007). "Chemical extractive compounds determining the brown-rot decay resistance of teak wood," Holz Roh Werkst 65(2), 121-124. DOI: 10.1007/s00107-006-0127-7

Vazquez, I. B., Fente, C., Franco, C. M., Vazquez, M. J., and Cepeda, A. (2001). "Inhibition effects of eugenol and thymol on Penicillium citrinum strains in culture media and cheese," International Journal of Food Microbiology 67(1-2), 157-163. DOI: 10.1016/S0168-1605(01)00429-9 
Windeisen, E., Klassen, A., and Wagener, G. (2003). "On the chemical characterization of plantation teakwood from Panama," Holz Roh-Werkst 61(6), 416-418. DOI: 10.1007/s00107-003-0425-2

Zambonelli, A., Zechini, A., Aulerio, D., Bianchi, A., and Albasini, A. (1996). "Effects of essential oils on phytopathogenic fungi in vitro," Journal of Phytopathology 144(910), 491-494. DOI: 10.1111/j.1439-0434.1996.tb00330.x

Article submitted: February 27, 2019; Peer review completed: April 12, 2019; Revised version received and accepted: April 18, 2019; Published: April 22, 2019.

DOI: $10.15376 /$ biores.14.2.4489-4511 